\title{
Quantized conductance in an atom-sized point contact
}

Olesen, L.; Laegsgaard, E.; Stensgaard, I.; Besenbacher, Flemming; Schiøtz, Jakob; Stoltze, Per; Jacobsen, Karsten Wedel; Nørskov, Jens Kehlet

Published in:

Physical Review Letters

Link to article, DOI:

10.1103/PhysRevLett.72.2251

Publication date:

1994

Document Version

Publisher's PDF, also known as Version of record

Link back to DTU Orbit

Citation (APA):

Olesen, L., Laegsgaard, E., Stensgaard, I., Besenbacher, F., Schiøtz, J., Stoltze, P., Jacobsen, K. W., \& Nørskov, J. K. (1994). Quantized conductance in an atom-sized point contact. Physical Review Letters, 72(14), 2251-2254. https://doi.org/10.1103/PhysRevLett.72.2251

\section{General rights}

Copyright and moral rights for the publications made accessible in the public portal are retained by the authors and/or other copyright owners and it is a condition of accessing publications that users recognise and abide by the legal requirements associated with these rights.

- Users may download and print one copy of any publication from the public portal for the purpose of private study or research.

- You may not further distribute the material or use it for any profit-making activity or commercial gain

- You may freely distribute the URL identifying the publication in the public portal

If you believe that this document breaches copyright please contact us providing details, and we will remove access to the work immediately and investigate your claim 


\title{
Quantized Conductance in an Atom-Sized Point Contact
}

\author{
L. Olesen, E. Lægsgaard, I. Stensgaard, and F. Besenbacher \\ Center for Atomic-Scale Materials Physics and Institute of Physics and Astronomy, Aarhus University, \\ DK 8000 Aarhus C, Denmark \\ J. Schiøtz, P. Stoltze, K. W. Jacobsen, and J. K. Nørskov \\ Center for Atomic-scale Materials Physics and Physics Department, Technical University of Denmark, \\ DK 2800 Lyngby, Denmark
}

(Received 22 December 1993)

\begin{abstract}
We present direct measurements at room temperature of the conductance of a point contact between a scanning tunneling microscope tip and $\mathrm{Ni}, \mathrm{Cu}$, and $\mathrm{Pt}$ surfaces. As the contact is stretched the conductance jumps in units of $2 e^{2} / h$. Atomistic simulations of the stretch of the contact combined with calculations of the conductance using the Landauer formula show that the observed behavior is due to the quantization of the transverse electron motion in a contact which contains between one and ten atoms.
\end{abstract}

PACS numbers: $73.40 . \mathrm{Cg}, 61.16 . \mathrm{Ch}$

Within the last few years a large number of experiments have shown unique electron transport properties of narrow $(\sim 1 \mu \mathrm{m})$ constrictions formed in GaAs-AlGaAs heterostructures [1]. One example is the quantization of the conductivity in units of $2 e^{2} / h$ [2] which has been interpreted using the Landauer formula as a consequence of the quantization of the electron motion perpendicular to the conductor $[3,4]$. The observation of these effects is limited to temperatures smaller than the energy spacing between the perpendicular modes. The mesoscopic size of the constrictions formed in semiconductor heterostructures restricts the temperatures where these effects are observable to be less than a few $\mathrm{K}[1]$.

When the tip of a scanning tunneling microscope (STM) is brought into contact with a metal surface a point contact is formed with very small cross section [5]. The contact can be thought of as an atomic size constriction in which the level spacing would be drastically larger than for the semiconductor mesostructures. Quantization of the conductance should therefore be observable at much higher temperatures. Recently, STM point contact measurements have shown jumps in the tunnel current as the contact has been stretched [6,7]. Similar observations have been reported based on the mechanically controllable break junction technique [8]. These observations may be an illustration of the quantized conductivity in a very narrow constriction, but the situation is less clear than in the semiconductor heterostructure case. It has been found that for some metals ( $\mathrm{Al}$ and $\mathrm{Ni}$ ) the unit of quantization of the conductance is $2 e^{2} / h$ and for others $(\mathrm{Pt})$ it is not [8]. Also the interpretations of the quantization usually differ, since most authors ascribe the jumps in conductance during stretching to abrupt changes in the cross-sectional area of the contact connected with the formation of new crystal planes in the contact region [9].

In the present Letter we demonstrate that in point contacts of atomic dimensions made in an STM, the con- ductance is quantized in units of $2 e^{2} / h$ even at room temperature. We measure the current and the voltage over the tip-surface junction simultaneously under well controlled ultrahigh vacuum (UHV) conditions on single crystal surfaces. The directly measured conductance $G$ shows jumps in units of $2 e^{2} / h$ as the contact is stretched for all the metals $(\mathrm{Ni}, \mathrm{Cu}, \mathrm{Pt})$ studied. Combining these observations with an atomistic simulation of the contact and a model calculation of the conductance based on a Landauer-type theory, we show that the jumps in $G$ are a direct consequence of the quantization of the transverse wave vector in a simple one dimensional contact rather than being directly related to sudden jumps in the width of the contact. In particular, we are able to model not only the quantized nature of the conductance during stretching of the contact, but also the absolute magnitude of the stretch per quantum level showing that the dimensions of the contact in the experiment and in the simulation are the same. These experiments thus provide the characteristics of ultimately small contacts consisting of only one to ten atoms.

The experiments, all carried out at room temperature on single crystal $\mathrm{Cu}, \mathrm{Ni}$, and $\mathrm{Pt}$ surfaces, were performed with a compact, high stability UHV STM [10] by recording simultaneously both the current and the voltage in the tip-surface junction during the tip approach and retreat from the sample surface. Typical experimental results for $\mathrm{Ni}(100)$ are depicted in Fig. 1. Prior to the tip indentation, the clean $\mathrm{Ni}(100)$ surface is scanned, and atomic resolution is obtained with typical tunneling parameters $I_{t} \sim 2-5 \mathrm{nA}$ and $V_{t} \sim 2-5 \mathrm{mV}$, that is, the tunnel conductance is of the order $1 \mu \mathrm{S}$. This corresponds to a tip height of 2-3 $\AA$ and in Fig. 1 this tunneling height defines the reference point $(z=0)$ for the subsequent tipindentation sequence. At a chosen point in the STM $x-y$ scan the feedback loop is opened, the tip is withdrawn $\sim 15 \AA$ and subsequently driven $\sim 20 \AA$ towards the surface at a rate of $\sim 1000 \AA / \mathrm{s}$. As seen from Fig. 1(a) 


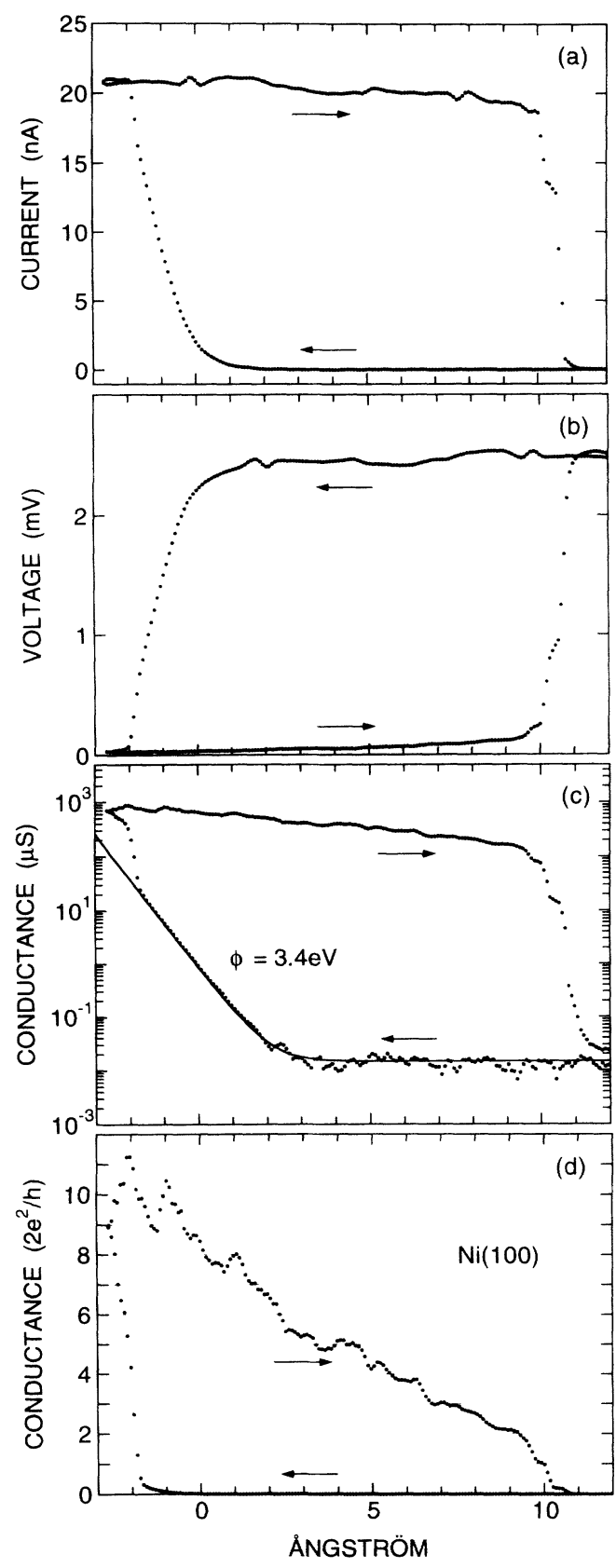

FIG. 1. Tip indentation curves for $\mathrm{Ni}(100)$ showing the current (a), voltage $\left(V_{j}\right)(\mathrm{b})$, and conductance (c) and (d) across the junction. During the approach the conductance is seen to follow an exponential increase all the way to point contact, yielding a constant effective barrier height. This observation is in disagreement with previous studies of $I$ versus $z$, where a varying barrier height was found. This will be discussed further elsewhere.

the current in the junction increases with decreasing tip surface distance until suddenly a point contact between the tip and the surface is formed and the current saturates at $\sim 20 \mathrm{nA}$. Subsequently the depth-time sequence is reversed, that is, the tip is withdrawn from the sur- face again at a rate of $1000 \AA / \mathrm{s}$, and the point contact breaks, causing a dramatic drop in the current to a value in the tunneling regime. Finally, the tip is brought back to the reference point $(z=0)$, the feedback loop is closed, and the scanning is continued. The total time for the tip-indentation/retraction sequence is $\sim 30 \mathrm{~ms}$.

It is most important for these studies to realize that the actual voltage across the junction $\left(V_{j}\right)$ is not at all constant but decreases dramatically as the tip is driven towards the surface; see Fig. 1(b). The reason for this is the finite input resistance $(120 \mathrm{k} \Omega)$ of our current preamplifier, and the applied tunnel voltage $\left(V_{t}\right)$ is divided between this input resistance and the resistance of the STM junction. In order to measure $V_{j}$, an additional low noise and high input impedance amplifier is connected directly across the junction. The low noise level $\sim 3 \mu \mathrm{V}$ rms allows an accurate determination of $V_{j}$ corresponding to a resolution of about $100 \Omega$ of the junction resistance. This scheme allows for both high and low impedance measurement of the junction over 6 orders of magnitude. Figure 1 (c) shows the ratio between the current and the voltage in the junction, that is the variation in the conductance $G$. During the approach of the tip to the surface, $\ln G$ varies linearly with tip sample separation as expected in the tunneling regime. At a distance of $z=-2 \AA$ the tip snaps into point contact with the surface causing a sudden jump in $G$. A Ni contact neck is formed and when the tip is withdrawn, the contact neck stretches. During this stretching it is observed that the conductance is quantized in units of $2 e^{2} / h(77.5 \mu \mathrm{S})$, until the contact finally breaks at $z \sim 10 \AA$ [Figs. 1(c) and 1(d)].

When the scanning is continued after point-contact formation and disruption, a $2-5 \AA$ high and 10-50 $\AA$ wide protrusion is found on the Ni surface. Results such as those shown in Fig. 1 are reproducible only after several indentations have been performed. In this case the original $\mathrm{W}$ tip is probably covered with $\mathrm{Ni}$ and thus the results are interpreted as an indentation of a $\mathrm{Ni}$ tip into a $\mathrm{Ni}(100)$ surface. For each new indentation $G$ is always quantized in units of $2 e^{2} / h$, but exactly which quanta of $2 e^{2} / h$ are observed differs from indentation to indentation.

Figure 2 shows conductance curves for clean $\mathrm{Cu}(100)$, $\mathrm{Pt}(100)$, and oxygen covered $\mathrm{Cu}(100)$ surfaces. In all cases the conductance $G$ is quantized in units of $2 e^{2} / h$. A typical length ( $z$ displacement) per quantum step is $\sim 1.3 \AA$ for $\mathrm{Ni}, \sim 1 \AA$ for $\mathrm{Cu}, \sim 0.7 \AA$ for $\mathrm{Pt}$, and $\sim 0.8 \AA$ for $\mathrm{O} / \mathrm{Cu}(100)$.

In our modeling of these experiments we notice from Fig. 1 that during the stretching of the contact a wire must be pulled between the tip and the surface, since the jumps in conductivity are observed after the contact has been stretched more than $10 \AA$ longer than the original length of the tip. We therefore concentrate on the thinning of the neck during the retraction of the STM tip, by performing a series of Langevin dynamics simulations of a metallic wire as a function of its length using the 


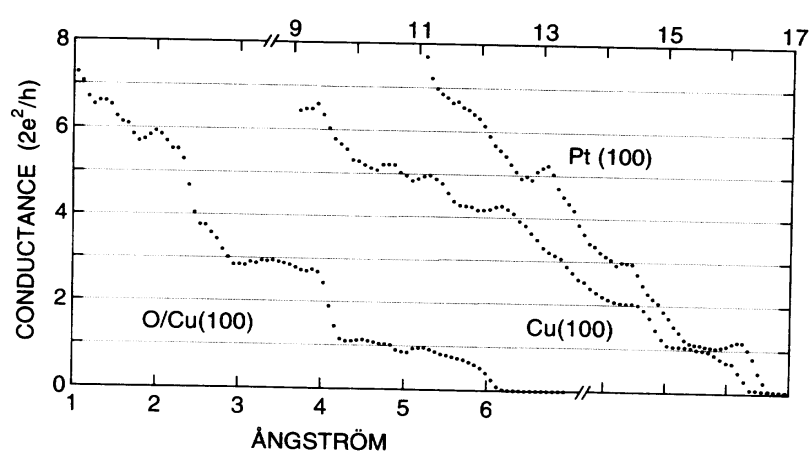

FIG. 2. Conductance curves for $\mathrm{O} / \mathrm{Cu}(100), \mathrm{Cu}(100)$, and $\operatorname{Pt}(100)$; only the quantized parts of the curves are shown [see Fig. 1(d)]. While the stretching length of the neck for clean $\mathrm{Cu}$ and $\mathrm{Pt}$ is comparable to the $\mathrm{Ni}$ case, it is considerably shorter for the oxygen covered surface, presumably due to a smaller diffusivity here.

interaction potential derived from the effective medium theory [11]. We have concentrated on a $\mathrm{Ni}(100)$ wire with a starting cross section of $3 \times 4$ atoms and which is 16 atoms long. If we start with a thicker wire then the last part of the simulation will be similar to the results shown here.

The positions of the atoms in the wire were first optimized by steepest descent minimization. The data were then recorded while the wire was strained by keeping the lower three layers fixed, while the upper three layers were moved $15 \AA$ over 216 ps at $300 \mathrm{~K}$. The strain rate is $5.4 \% / \mathrm{ps}$ equivalent to a speed of $7.2 \mathrm{~m} / \mathrm{s}$. We obtained similar results at $600 \mathrm{~K}$, using a strain rate of $1.6 \mathrm{~m} / \mathrm{s}$ and in simulations using simulated annealing over limited times and at moderate temperatures to find local equilibrium configurations. We have also done simulations for $\mathrm{Cu}$ and $\mathrm{Pt}$ with very similar results.

From the simulations, the minimum cross-sectional area of the wire can be extracted as a function of the length. This is shown in Fig. 3. The area changes in rather well defined jumps associated with a series of mechanical instabilities as suggested by Landman et al. [12] and Todorov and Sutton [9]. We find, in agreement with Landman et al. [12], that the instabilities are associated with dislocation formation.

To calculate the conductance of the nanowire we employ the theory of Landauer [3] and Büttiker et al. [4]. They have shown that the conductance of a onedimensional conductor with a barrier connecting two reservoirs is

$$
G=\frac{2 e^{2}}{h} \sum_{i}\left(1-R_{i}\right), \quad R_{i}=\sum_{j} R_{i j}
$$

where $R_{i j}$ is the probability that an electron that enters channel $i$ is reflected in channel $j$. Here we have defined the conductance as the ratio of the current through the conductor and the voltage between the reservoirs.

When the electrons are moving in a very narrow constriction, the electron motion becomes quantized in the

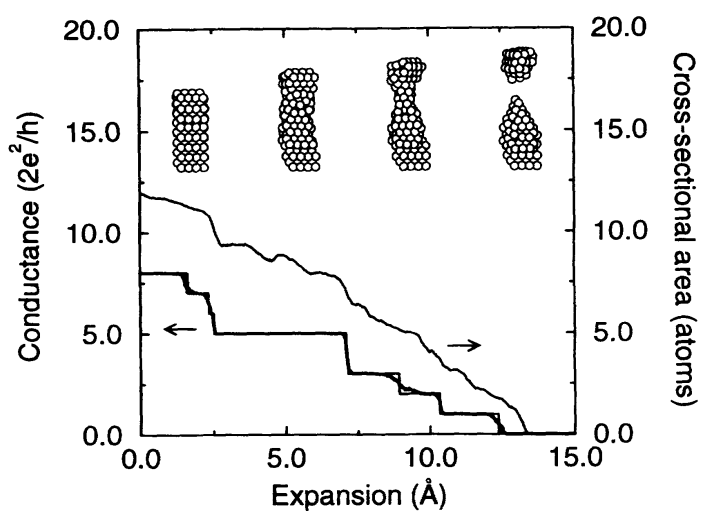

FIG. 3. The calculated minimum cross-sectional area of the wire as a function of the elongation. Also shown is the calculated conductance with (thick curve) and without tunneling included. In the simulations, thermal noise has been reduced by the formation of a running average over a $2.7 \mathrm{ps}$ interval. Insets show snapshots of the nanowire along the stretching process. The area of one atom is $6.27 \AA^{2}$.

transverse directions. We assume a narrow rectangular conductor in the $z$ direction limited by infinite potential walls in the $x-y$ plane, so that the potential is infinite in the region outside $-\frac{L_{x}(z)}{2}<x<\frac{L_{x}(z)}{2}$ and $-\frac{L_{y}(z)}{2}<y<\frac{L_{y}(z)}{2}$. We also assume that the dimensions in the $x-y$ plane vary slowly with $z$ (the adiabatic limit), then the Schrödinger equation can be solved in the $x-y$ plane, and the electron motion along the conductor is governed by a one-dimensional Schrödinger equation with the effective potential

$$
V_{n_{x} n_{y}}(z)=\frac{\hbar^{2} \pi^{2}}{2 m}\left(\frac{n_{x}^{2}}{L_{x}(z)^{2}}+\frac{n_{y}^{2}}{L_{y}(z)^{2}}\right) .
$$

Here $L_{x}$ and $L_{y}$ denote the dimensions of the conductor in the $x$ and $y$ directions. Each channel thus sees a different potential barrier, and if the spacings between the potential barriers are large compared to the region, where tunneling through the barrier is important, we will always have an integer number of open channels. We will thus see a conductance of $N_{\text {open }}\left(2 e^{2} / h\right)$, where $N_{\text {open }}$ is the number of states for which the Fermi energy is larger than the maximum value of the potential $V_{n_{x} n_{y}}$. For a given form of the constriction the latter is given by the minimum cross-sectional area through $L_{x}$ and $L_{y}$.

A more detailed theoretical description will have to include tunneling and reflection at the barrier and coupling between different channels due to nonadiabatic effects. Assuming that the constriction shape $L(z)$ is Gaussian with a width of $a$ and a maximum amplitude $\Delta L$ and a minimum value of $L_{0}$ the energy window in which tunneling is important is $\Delta E=\left(2 \hbar^{2} / 2 m\right) \sqrt{\frac{\Delta L}{a^{2} L_{0}^{3}}}$. From the simulations we find that $a$ is typically $15 \AA$, which means that tunneling only plays a minor role for the geometry considered here. This can be seen in Fig. 3 which contains the calculated conductance both without and with 
tunneling included [13]. The coupling between the different channels can also be estimated [14]. If $(d L / d z) \lambda<L$, where $\lambda$ is the de Broglie wavelength of the scattering electron, then the coupling is negligible. This inequality is well obeyed for the form of $L(z)$ deduced from the simulation. We have checked this and the description of the tunneling given above by performing full coupled channel calculations of the conductance.

The simulations shown in Fig. 3 describe the experimental results of Fig. 2 in several ways. First, the quantized nature of the conductance is clearly seen. Second, the typical step size is the same, that is, most steps are one or two units of $2 e^{2} / h$ (as opposed to the simulations of Todorov and Sutton [9]), and, third, the length scale of the stretch per quantum unit is the same. The latter point clearly demonstrates that the cross section of the nanowire in the simulation and the contact in the experiment are the same, and thus shows that the point contact in the experiment consists of between one and ten atoms.

For the neck diameters of 5-10 $\AA$ seen in our simulations a typical energy spacing between the transverse levels is of the order $0.2-1 \mathrm{eV}$ or several thousand $\mathrm{K}$. In comparison the same energy spacing in a $250 \mathrm{~nm}$ constriction in a semiconductor device is $0.1 \mathrm{meV}$ corresponding to $1 \mathrm{~K}$. Temperature effects on the quantization of the conductivity in the STM contacts are therefore expected to be small at least to the melting point of the metals involved.

Although the model we use is quite different from the one-band tight-binding model used by Todorov and Sutton [9] to calculate the conductance, the result is essentially the same. We also find that the conductance per atom is of the order a little more than half a unit of $2 e^{2} / h$ and that the cross-sectional area of the contact decreases quite abruptly as the wire is stretched. The additional feature of our modeling is that it allows us to understand the origin of the quantized conductance better. It is clear from the analysis above that the quantization is not directly related to mechanical instabilities giving rise to the jumps in the contact area as suggested by Todorov and Sutton [9]. Such a correlation might be suggested from Fig. 3, but from Eq. (2) it is clear that the quantized nature of the conductance as a function of the stretch of the contact will exist for any decreasing transverse length scale of the neck irrespective of the exact nature of the mechanism behind it.

The simple rectangular model leading to Eq. (2) suggests that the form of the cross section of the neck will define the exact quantization of the conductance. This first of all suggests that the sequence of quanta should differ from one experiment to the next because the form of the neck is the result of diffusion processes which cannot be controlled on the atomic scale. This is in clear accordance with our observations. It also suggests that in some special cases where the two length scales of the neck are approximately equal new degeneracies should occur. Such examples are indeed found in our experiments. In Fig. 2 we show an indentation curve on a $\mathrm{O} / \mathrm{Cu}(100)$ surface. In this case we observe the quantum levels of $N_{\text {open }}=1,3,4,6,8 \ldots$, which is exactly the series expected for a quadratic channel.

In summary, we have given clear experimental evidence for a quantized conductance in metallic point contacts formed between an STM tip and a metal surface at room temperature. By combining the experimental observations with dynamical modeling of the stretching of the contact and a Landauer-type theory for the conductance we show that the quantization of the conductance is due to the quantization of the transverse electron motion in the contact consisting of between one and ten atoms only.

Center for Atomic-Scale Materials Physics is sponsored by the Danish National Research Foundation. We gratefully acknowledge discussions with P. Hedegaard and A. P. Jauho as well as the technical support by F. Søeberg and E. Søndergaard.

[1] For a recent review, see, e.g., C. W. J. Beenakker and H. van Houten, in Solid State Physics, edited by H. Ehrenreich et al. (Academic, New York, 1991), Vol. 44, p. 1.

[2] B. J. van Wees, H. van Houten, C. W. J. Beenakker, J. G. Williamson, L. P. Kouwendhoven, D. van der Marel, and C. T. Foxton, Phys. Rev. Lett. 60, 848 (1988); D. A. Wharam, M. Pepper, H. Ahmed, J. E. F. Frost, D. G. Hasko, D. C. Peacock, D. A. Ritchie, and G. A. C. Jones, J. Phys. C 21, L209 (1988).

[3] R. Landauer, IBM J. Res. Dev. 1, 223 (1957); Philos. Mag. 21, 863 (1970).

[4] M. Büttiker, Y. Imry, R. Landauer, and S. Pinhas, Phys. Rev. B 31, 6207 (1985).

[5] N. D. Lang, A. Yacoby, and Y. Imry, Phys. Rev. Lett. 63, 1499 (1989).

[6] N. Agrait, J. C. Rodrigo, and S. Vieira, Phys. Rev. B 47, 12345 (1993).

[7] J. I. Pascual, J. Méndez, J. Gómez-Herrero, A. M. Baró, N. García, and Vu Thien Binh, Phys. Rev. Lett. 71, 1852 (1993).

[8] J. M. Krans, C. J. Muller, I. K. Yanson, Th. C. M. Govaert, R. Hesper, and J. M. van Ruitenbeek, Phys. Rev. B 48, 14721 (1993).

[9] T. N. Todorov and A. P. Sutton, Phys. Rev. Lett. 70, 2138 (1993).

[10] C. Klink, L. Olesen, F. Besenbacher, I. Stensgaard, E. Lægsgaard, and N. D. Lang, Phys. Rev. Lett. 71, 4350 (1993).

[11] K. W. Jacobsen, J. K. Nørskov, and M. J. Puska, Phys. Rev. B 35, 7423 (1987).

[12] U. Landman, W. D. Luedtke, N. A. Burnham, and R. J. Colton, Science 248, 454 (1990).

[13] In the free-electron description of the $\mathrm{Ni}$ electrons we implicitly only describe the $s$ electrons which have a Fermi energy of $7.41 \mathrm{eV}$.

[14] A. Yacoby and Y. Imry, Phys. Rev. B 41, 5341 (1991). 